\title{
Civil Society in Albania and the Impact of EU in Its Development
}

\author{
Arian Dedej, PhD Candidate
}

Lecture at "Aleksander Moisiu" University

Email: ariandedej@hotmail.com

\section{Dr. Fatbardha Kadiu}

Lecture at "Aleksander Moisiu" University

Email: fatbardhakadiu@yahoo.com

Elona Dollaku

Email: elona.dollaku@gmail.com

Doi:10.5901/mjss.2014.v5n23p1965

\begin{abstract}
Albania has experienced one of the most brutal dictatorships in Eastern Europe. For 20 years, it is undergoing a period of transition towards democracy and the European Union. One of the most important criteria to meet these goals is the development and strengthening of the civil society, in whose heart are the non-profit organizations. History of NGOs and Civil Society in Albania cannot be compared with that of other countries, even the communist ones. This phenomenon has affected the organization and the development of the Civil Society and democracy in Albanian. Experience shows that actors and institutions of civil society can play an important and crucial role in the strengthening of democracy as well as in the integration process. Citizens are beginning to have concepts and believe in the democracy and integration in the European Union. This process should not be imposed by the political class, which means in a top-down direction, but quite the opposite. In this way, the civil society being informed and having access to policy making process, will help in formulating realistic policies in favor of integration. Albania's integration process should be inclusive and active. For this, it is important and necessary the irreplaceable role of the Civil Society. To understand the opinion of civil society and its position in relation to the role of NGOs I have compiled a questionnaire, the analysis of which has helped me to reach conclusions more easily.
\end{abstract}

Keywords: Civil society, NGOs, European Union

\section{L'approccio dell'Unione Europea Verso La Societa' Civile}

La democrazia partecipativa è ormai parte integrante del modello europeo di società. II Trattato di Lisbona sancisce la complementarità tra democrazia rappresentativa e democrazia partecipativa, contenute rispettivamente negli articoli $10 \mathrm{e}$ 11. Inoltre, l'articolo 10, paragrafo 3 , del Trattato conferisce ai cittadini «il diritto di partecipare alla vita democratica dell'Unione», e, precisando che «le decisioni sono prese nella maniera il più possibile aperta e vicina ai cittadini», rinvia alla necessità di applicare il principio di sussidiarietà. La partecipazione diviene quindi un diritto dei cittadini e la sussidiarietà un elemento portante della democrazia partecipativa (CESE, 2011).

Articolo 11

1. Le istituzioni danno ai cittadini e alle associazioni rappresentative, attraverso gli opportuni canali, la possibilità di far conoscere e di scambiare pubblicamente le loro opinioni in tutti i settori di azione dell'Unione.

2. Le istituzioni mantengono un dialogo aperto, trasparente e regolare con le associazioni rappresentative e la società civile.

3. Al fine di assicurare la coerenza e la trasparenza delle azioni dell'Unione, la Commissione europea procede ad ampie consultazioni delle parti interessate.

4. Cittadini dell'Unione, in numero di almeno un milione, che abbiano la cittadinanza di un numero significativo di Stati membri, possono prendere l'iniziativa d'invitare la Commissione europea, nell'ambito delle sue attribuzioni, a presentare una proposta appropriata su materie in merito alle quali tali cittadini ritengono necessario un atto giuridico dell'Unione ai fini dell'attuazione dei Trattati. Le procedure e le condizioni necessarie per la presentazione di una iniziativa dei cittadini sono stabilite conformemente all'articolo 24 , 
primo comma del Trattato sul funzionamento dell'Unione europea.

\section{La Storia della Societa' Civile Albanese}

L'esperienza dei paesi ex communisti come Germania Orientale, Polonia e Ungheria, ha dimostrato che la miglior risposta contro un regime totalitario è il rafforzamento del coraggio civico dei cittadini in una società e delle loro istituzioni libere. Anche l'Albania si è confrontata con una situazione del genere, ma qui i sviluppi non erano identici con quelli della maggior parte dei paesi ex-comunisti. In Albania il cambiamento del sistema politico e l'inizio della democrzia nel dicembre del 1990 sono stati possibili attraverso il movimento degli studenti, il quale si è trasformato poi in un movimento popolare (Krasniqi, 2004).

Diversamente dagli altri paesi ex comunisti dell'Europa dell'Est, nei quali già dagli anni '70 si intravvedevano dei gruppi che rappresentavano interesi particolari, in Albania non c'è stata alcun azione di qualche gruppo indipendente fino alla fine del regime communista, appunto nel dicembre del 1990. Prima di questa data non esisteva nessuna zona intermedia tra lo Stato e la società, perche' il partito communista controllava tutte i campi della società. Questo patrimonio del regime comunista ha fatto sì che all'Albania mancasse una tradizione per quanto riguardava i movimenti sociali, e ha creato ostacoli per la costruzione di una societa civile libera e viva, la quale è un elemento importante per il considolamento della democrazia (Biberaj, 2000).

L'organizzazione della società civile è un settore indispensabile in una societa democratica. Anche se queste società si sono create dopo la caduta del comunismo, esse sono diventate importanti per numero ed attività. Lo svillupo continuo democratico dipende dalla forza e dall'organizzazione di questo settore importante, ma forse anche dalla collaborazione attraverso le organizzazioni della societa civile e gli altri settori della società albanese (ORT/USAID, 1999).

A parte le organizzazioni della società civile e le organizzazioni dei diritti fondamentali degli uomini e quello delle donne, il periodo tranzitorio post-comunista ha aiutato nello sviluppo di nuove forme di organizzazione anche come gruppi di esperti per le politiche, conosciuti con il nome Think Tank . il primo di essi che si è creato nel 1992 era il centro albanese per ricerche economiche. Altri campi che hanno attirato l'atenzione sono la risoluzione degli confliti, specie dopo la rinascita del kanun e delle faide, lo sviluppo dell'ambiente e quello economico, gioventù e sport. Quasi il 30\% di queste organizzazioni della società civile si sono registrate nel primo periodo post communista (IDM-CIVICUS, 2010).

Lo svilupo del paese è stato messo in seria discussione dalla crisi del 1997 causata dal crollo delle piramidi. Questo evento ha avuto une impatto più che negativo nella vita economica, politica e della societa del paese. La situazione si è complicata ancor di più dalla guerra del Kososvo nel 1999 e del fatto che più di une milione di kosovari sono stati costretti a rifugiarsi in Albania. Questi eventi hanno portato allo svilupo di una grande comunità di organizzazioni della societa civile in Albania, le quali trattano delle questioni che vanno dalla protezione dei diritti delle donne fino a quelle contro le mine (Report, 1999). Quasi il 49\% delle Organizazioni delle societa civile registrate in Albania si sono create dal periodo 1997 fino al 2001 (CENTER, 2009).

II nuovo quadro legislativo sulle Organizzazione Non Governative, o anche ONP (organizzazioni non profit), è del 2001, il quale è stato costruito in modo partecipativo attraverso un consensus tra le parti coinvolte, (rappresantanti del governo, esperti internazionali dove il ruolo piu importante l'ha avuto la International Center for Not-for-Profit Law, rappresentanti della Societa Civile e della OSCE) praticamente per tutte le questioni.

L'Attivita della Societa Civile dopo il 2005 si e' caratterizata dopo i nuovi sviluppi politici quyestione che si e' reflesso anche nel modo in cui che si e' percepita la Societa Civile. Questo periodo si è caratterizzato per un aumento degli attori della societa civile che sono entrati in politica, facendo svanire i confini dei due settori nell'opinione pubblica. Di consenguenza molti sostenitori finanziari delle organizzazione della societa civile hanno diminuito i loro fondi che di conseguenza hanno diminuito la cornice geografica del terzo settore (IDM-CIVICUS, 2010).

Indipedentemente dall'indebolimento del terzo settore in Albania, iniziative positive degli ultimi tempi sono state fatte da parte del governo albanese per il miglioramento della cornice legislativa sulla società civile. Nel ottobre del 2007, il consiglio dei ministri ha deciso di creare una voce aposita ne Budget dello Stato "per sostenere la societa civile". Nel marzo del 2009, il parlamento albanese ha approvato la legge "Sull'organizzazione e il funzionamento dell'Agenzia di Supporto della Societa Civile" e delle procedura che si deve attuare per la distribuzione degli fondi a sostegno della Societa Civile. Altri passi si sono intrapresi da parte di organizzazioni internazionali per potenziare la Societa Civile in Albania. Uno dei risultati più importanti di queste iniziative e la consultazione su larga scala e l'approvazione della Carta della Societa Cicile nel 2009. Indipendentemente da questi sviluppi, pochi erano quei ministeri e dipartimenti che hanno creato i meccanismi necessari per il convolgimento della Societa Civile e la sua abilità amministrativa, ancora oggi il lavoro svolto su questa direzione e ancora molto piccolo (IDM-CIVICUS, 2010). 


\section{II Supporto dell'Unione Europea nel Terzo Settore in Albania}

La creazione della Agenzia di Supporto della Societa Civile da parte del governo e la sua perfomance in qusti ultimi anni dimostra che anche lo stato sta facendo lo stesso sbaglio come la maggior parte dei donatori, assicurandosi finanze in piu per le OSC, ma non aiutando realmente nelle condizioni finanziarie e altre condizioni che porterebbero in stabilita del settore. La mobilitazione dei fondi nazionali e il filantropismo sono solo una parte dei "compiti del governo". Nel contesto degli effetti nazionali (specificamente nelle zone periferiche del paese) della crisi globale, i partenariati efettivi per i costi della societa civile, non sono stati presi in considerazione dallo stato. In questo contesto, la fondazione della ASSC è un buon passo ma che serve per di piu per colmare il gap creatosi dall'allontanamento degli donatori come anche rispondersi alle necessità delle organizzazioni della società civile locali le quali non possono "mandar giu" le regole manageriali degli programi sostenuti dall UE, il piu grande donatore per Societa Civile in Albania (IDM, 2013).

Nel 2008 la Commissione Europea ha aprovato uno strumento per sostenere la societa civile nei paesi candidati e potenzialmente candidati: la "Civil Society Facility". Questo strumento si è creato nell'ambito del programma IPA per promuovere lo sviluppo e il dialogo con la società civile. Dal 2007 al 2013 l'Albania ha percepito piu di 595 milioni di euro.

\begin{tabular}{|l|c|c|c|c|c|c|c|}
\hline Component & $\mathbf{2 0 0 7}$ & $\mathbf{2 0 0 8}$ & $\mathbf{2 0 0 9}$ & $\mathbf{2 0 1 0}$ & $\mathbf{2 0 1 1}$ & $\mathbf{2 0 1 2}$ & $\mathbf{2 0 1 3}$ \\
\hline $\begin{array}{l}\text { Transition Assistance and } \\
\text { Institution Building }\end{array}$ & $54,318,790$ & $65,237,756$ & $71,360,000$ & $84,200,000$ & $84,301,650$ & $84,290,995$ & $84,682,966$ \\
\hline Cross-border Co-operation & $6,681,210$ & $8,582,244$ & $9,822,921$ & $9,973,173$ & $10,126,636$ & $10,283,169$ & $10,666,232$ \\
\hline Total & $61,000,000$ & $73,820,000$ & $81,182,921$ & $94,173,173$ & $94,428,286$ & $94,574,164$ & $95,349,198$ \\
\hline
\end{tabular}

IPA-Albania

CSF si concetra su tre campi d'azione: 1-sostenere per sviluppare le iniziative e le capacità della SC locale, per rafforzare il suo ruolo. 2-programmi diretti per favorire i contatti con i giornalisti, giovani politici, leader degli sindacati, insegnanti e i rapresentatnti delle ONG con le istituzioni dell'UE. 3- sostenere la costruzione dei partnetariati e lo sviluppo degli network tra le ong e le imprese, sindacati e i gruppi di profesioniti, sia a livello nazionale sia a livello dell'UE. (CSF, 2008)

Dal 2007, l'UE ha contribuito ogni anno con quasi 600.000 euro per le attivita degli cittadini sui diritti umani attraverso lo strumento europeo per la Democrazia e i diritti umani. Negli ultimi 7 anni, UE ha sostenuto le attivita delle ONG con più di 12.500 .000 euro per promuovere il rispetto dei diritti umani e il coinvolgimento sociale, la guerra contro la corruzione, la salvaguardia del patrimonio culturale e la protezione dell'ambiente.

Anche nel contesto dei programmi di sostentamento dell'UE il governo deve prendersi le sue responsabilità e vedere i paesi vicini. Per esempio il governo macedone si è impegnato ad offrire il $20 \%$ del cofinaziamento per qualsiasi progetto delle ONG approvato negli schemi finanziari dell UE (IDM, 2013).

Per evolvere il ruolo della Societa Civile si deve rafforzare il dialogo che sembra molto debole e infruttuoso tra la Società Civile e il governo. II coinvolgimento della società civile nei processi legislativi, sia nel momento dell'elaborazione sia in quell del monitoraggio, è debole sia per l'efficacia delle riforme sia per il loro consenso.

Secondo il CESE l'attivita delle Organizzazioni Non Governative si deve rafforzare nelle zone rurali e meno svilupate dell'Albania, ma la società civile albanese rimane concentrata a Tirana e nei dintorni della capitale. (EESC, 2007)

Attualmente in Albania sono registrate 1951 ONG e solo 314 di loro si sono registrate presso ASSC, mentre 1637 hanno il loro centro in Tirana, cosa che deve far riflettere la società civile a spostare il suo baricentro nelle zone piu disagiate del paese. 


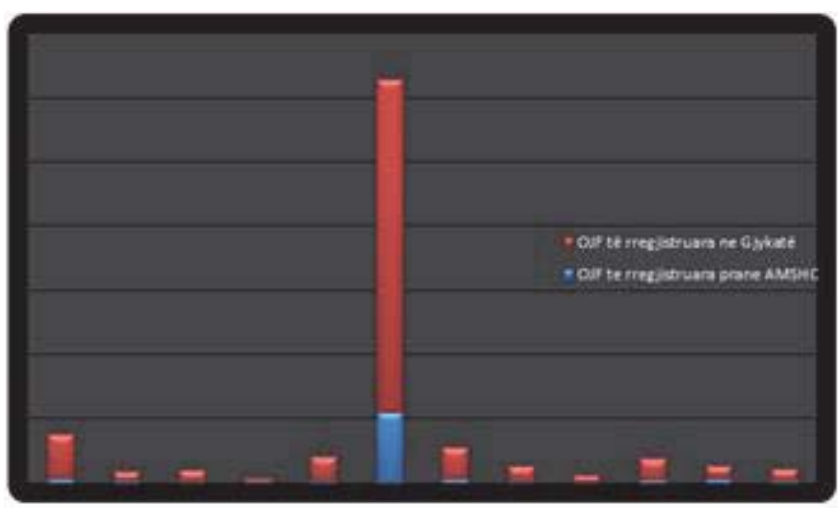

Source: Agjensia për Mbështetjen e Shoqërisë Civile

Nonostante il numero elevato delle organizzazioni della società civile, i cittadini albanesi secondo un questionario elaborato per questo studio sono ancora diffidenti della società civile. Loro pensano che la società civile abbia un ruolo fondamentale nel progresso del paese e nel suo cammino verso l'Unione Europea ma la società civile la deve smettere di essere agli ordini della politica, deve costruirsi la propia indipendenza cominciando da quella economica (EESC, 2007).

\section{Conclusioni}

La societa civile e' un settore molto importante di una societa' democratica e per questo motivo che l'UE vuole dare maggior peso politico passando da una democrazia rappresantativa in una democrazia partecipativa.

L'Albania che aspira a diventare un nuovo membro dell'Unione Europea sta cercando di rafforzare questo settore anche se tale obbiettivo rimane tuttora molto dificile. La societa' civile in Albania continua ad essere sotto il "controllo" della classe politica albanese.

Questa situazione avviene anche dal fatto storico della societa' civile in albania, la quale e' stata condizionata anche dal regime communista albanese uno dei piu chiusi e spietati al mondo.

Le organizzazioni della societa' civile in albania dalla sua parte deve cercare di essere piu indipendente e deve seguire i consigli del Comitato Economico Sociale Europea e spostarsi dalla capitale e dalle zone urbane verso le zone piu dissagiate del paese.

II contributo dell'Unione Europea e stato e continuera ad essere molto significativo ed importante nello sviluppo della societa' civile albanese quale paese che aspira che in un futuro diventi parte della grande famigla europea.

\section{Bibliografia}

Akademia e Shkencave e Shqipërisë "Historia e Popullit Shqiptar II" , Toena, Tiranë,2002.

Akademia e Shkencave e Shqipërisë "Historia e Popullit Shqiptar III",Toena, Tirane, 2007.

Akademia e Shkencave e Shqipërisë "Historia e Popullit Shqiptar IV", Toena, Tirane,2009

Alexis de Tocqueville, "La democrazia in America". UTET, Torino, 2007.

Andrew McLaughlin, Justin Greenwood, "The Management of Interest Representation in the European Union", JCMS: Journal of Common Market Studies 33 (1) 1995.

Baker Gideon, "The Taming of the Idea of Civil Society", Taylor \& Francis Ltd, Oregon 2004.

Banfield Edward, "The Moral Basis of a Backward Society", The Free Press. Glencoe 1958.

Biberaj Elezaj "Shqipëria në tranzicion-Rruga e vështirë drejt demokracisë", ORA, Tirane, 2000.

Bryant Christopher, "Civil Society and Incivility in Tatcherite Britain: What Are the Lessons?", Paper presented at the International Symposium on the Philosophy of Social Choice, Polish Academy of Science, Warsaw, 1990.

Burgio Alberto, "Gramsci storico. Una lettura dei Quaderni del carcere", La Terza, Bari, 2003.

Chandhoke Neera, "What the hell is 'civil society'?" opendemocracy, 17 March 2005. http://www.opendemocracy.net/democracyopen_politics/article_2375.jsp

Civil Society Development Center "Konceptet e Shoqerise Civile" ,CSDC, Durres, 2010.

Comaroff John, "Civil Society and the Political Imagination in Africa. Critical Perspectives" The University of Chicago Press, ChicagoLondon 1999.

Connor Robert, "The Idea of Civil Society"National Humanities Center, fevrier, 1998.

Dahrendorf Ralf, "Economic Opportunity, Civil Society, and Political Liberty", United Nations Research Institute for Social Development, 
Geneva 1995.

European Economic and Social Committee, "Enlargement Strategy and Main Challenges 2007-2008". Brussels COM(2007) 663 final: Communication from the Commission to the European Parliament and Council, Brussels, 2007.

European Commission, "An open and structured dialogue between the Commission and special interest groups". Brussels, 1992.

European Commission, "European Commission DG Enlargement to give financial support the development of civil society in the Western Balkans and Turkey" Civil Society Facility Partnership Programmers for Civil Society Organizations. Brussels, 2008.

European Commission, "Commission Discussion Paper. The Commission and Non Governmental Organizations: building a Stronger Partnership". Brussels 2000.

European Commission "Promoting the Role of Voluntary Organisations and Foundations in Europe", Brussels, 1997.

European Commission, "Report from the Commission on European Governance" Office for Official Publications, Luxembourg 2003/a.

European Commission, "European Governance - a White Paper", Brussels, 2001

European Commission Founding by country, Albania. Brussels: Enlargement, Instrument for Pre-accession Assistance (IPA), 2014.

Fischer Bernd J., "Mbreti Zog dhe perpjekja për stabilitet në Shqipëri" Tirane: Cabej, 2004.

Gojko Bežovan, Siniša Zrinšak \& Marina Vugec, "Civil Society in Croatia: Gaining Trust and Establishing Partnership ëith the state and other Stakeholders", CIVICUS-CERANEO, Zagreb, 2005.

Gori Luca, "L'Unione Europea e i Balcani Occidendtali, Rubbettino, Soveria Mannelli, 2007.

Greenwood Justin."Organized Civil Society and Democratic Legitimacy in the European Union". British Journal of Political Science 37 (02) , 2007, 333-57.

Guillermo O'Donnell, P. S. "Transitions from Authoritarian Rule. Tentative Conclusions about Uncertain", Johns Hopkins University Press, London, 1986.

Hardt Michael, "The Withering of Civil Society", Social Text, n. 45, 1995, f. 27-44.

Havel Vaclav, "Toward a Civil Society. Selected Speeches and Writings 1990-1994", Lidové Noviny, Prague, 1995.

Howell, Jude, and Jenny Pearce, Civil society \& development : a critical exploration. Boulder, Colo, Lynne Rienner Publishers, 2001/b.

Hurt, Stephen R "Co-operation and Coercion? The Cotonou Agreement between the European Union and ACP States and the End of the Lome Convention". Third World Quarterly 24 (1): 2003.

Human Development Promotion Centre" Third Sector Development in Albania: challenges and opportunities" Tirane, 2010.

Human Rights Watch Report 1999 bhttp://www.hrw.org/legacy/worldreport99/europe/albania.html.

Indeksi i Shoqërisë Civile për Shqipërinë, "Në kërkim të Qytetarëve dhe ndikimit”, IDM-CIVICUS-UNDP, Tirane 2010.

Instituti për Demokraci dhe Ndërmjetësim, "Policy brief " nr 1, Tirane Janar 2013,

Irwin Terence, "I princìpi primi di Aristotele" Largo A.Gemelli, Milano, 1996.

Krasniqi Afrim, "Shoqeria civile ne Shqiperi", GEER, Tirane, 2004.

Laurenti Roberto, "Politica"La Terza, Bari, 2007.

Leci Elmas, "Krime ne kohen e komunizmit" Instituti i Studimit të Problemeve të Sigurisë dhe Mbrojtjes, Tirane, 2007.

Lulli Francesca, "Microfinanza, economia popolare e associazionismo in Africa occidentale. Uno sguardo al femminile", Editori Riuniti, Roma 2008.

Mammarella Giussepe, Cacace Paolo, "Storia dell'Unione europea", Laterza, Bari 2004.

Maria Franco, II processo di adesione della Croazia all'Unione Europea: il ruolo della societa' civile quale attore di democatizzazione e di integrazione, Universita degli Sudi di Padova, 2008.

Marina Ottaway, Thomas Caothers, "Fundin Virtue" Brookings Institution Press, Washington 2000.

Ministria e Integrimit, "Versioni I Konsoliduar te Bashkimit Europian dhe Karta e te drejtave themelore e Bashkimit Europian, GIZ, Tirane, 2008.

Milo Paskal, "Bashkimi Evropian: Ideja, Integrimi, Identiteti, E ardhmja”, albPaper, Tiranë 2002.

Nwokedi Emeka "Governance and Democratization in West Africa" Cordesia, Dakar 1998.

Official Journal of the European Union "COUNCIL DECISION of 20 February 2006 on the principles, priorities and conditions contained in the Accession Partnership ëith Croatia and repealing Decision 2004/648/EC", L 55/30, 25.2.2006

Putnam Robert, "Making Democracy Work. Civic Traditions in Modern Italy" Princeton UP, Princeton, 1993. 